\section{Viscoelastic Properties of Polymer Melts from Equilibrium Molecular Dynamics Simulations}

\section{Suchira Sen, ${ }^{\dagger}$ Sanat K. Kumar, ${ }^{*}, \dagger$ and Pawel Keblinski ${ }^{\ddagger}$}

Isermann Department of Chemical and Biological Engineering, and Department of Materials Science and Engineering, Rensselaer Polytechnic Institute, Troy, New York 12180

Received October 2, 2003

Revised Manuscript Received December 6, 2004

Introduction. The dynamics of polymer chains are now generally very well understood in the pioneering frameworks of the Rouse model for short chains, and the reptation model for longer chains. ${ }^{1-5}$ While these theories yield well-known expressions for the variation of the diffusivity $D$ and viscosity $\eta$ with the chain length $N$, an open question is the chain length at which one crosses over from Rouse-like behavior to reptation. This length, termed the entanglement length, $N_{\mathrm{e}}$, is relatively easy to estimate experimentally, but has been hard to access in a simulation due to the fact that, up until recently, there was no satisfactory definition of an entanglement. 6,7

In numerical studies, most estimates of the entanglement length involve conducting simulations and examining dynamic quantities, such as the frequency dependent storage modulus. Extensive work has been done by Kremer and Grest ${ }^{8}$ to identify the onset of chain entanglement, with somewhat mixed results. Examination of the chain length dependence of chain diffusion yields a cross over from the Rouse scaling $\left(N^{-1}\right)$ to reptation scaling $\left(N^{-2}\right)$ behavior in the vicinity of chains of length 35. In contrast, estimates of storage modulus from nonequilibrium molecular dynamics simulations yield an entanglement chain length in the vicinity of 80.

We approach this problem with the tools of equilibrium molecular dynamics (MD). ${ }^{9}$ Equilibrium stress fluctuations which result naturally from these simulations directly provide estimates of the viscosity through the Green-Kubo equation, as suggested by Smith et al. ${ }^{10}$ The storage and loss modulus are also computed from the stress autocorrelation function, and the hints of a plateau are seen in the storage modulus. The plateau is the strongest indication of onset of reptation dynamics, and is seen for chain lengths of 80 and higher. Estimates of the entanglement length $N_{\mathrm{e}}$, provide a number closer to 30 , consistent with the first estimate provided by Kremer and Grest.

Simulation Model and Methods. The MD simulation employs a standard chain model. Interaction between nonbonded monomers are described by a shifted, purely repulsive Lennard-Jones (LJ) potential: $U(r)=$ $4 \epsilon\left[(\sigma / r)^{12}-(\sigma / r)^{6}\right]+\epsilon$ for $r<2^{1 / 6} \sigma$, and $U(r)=0$ for $r>$ $2^{1 / 6} \sigma$. Adjacent bonded monomers interact via a stiff FENE potential, in the form of $V_{\mathrm{FENE}}=-k\left(R_{0}{ }^{2} / 2\right) \ln (1$

\footnotetext{
$\dagger$ Isermann Department of Chemical and Biological Engineering, Rensselaer Polytechnic Institute.

$¥$ Department of Materials Science and Engineering, Rensselaer Polytechnic Institute.
}

Table 1. ${ }^{a}$

\begin{tabular}{ccccc}
\hline & $N=20$ & $N=40$ & $N=80$ & $N=120$ \\
\hline$\left\langle R_{\mathrm{e}}{ }^{2}\right\rangle\left(\sigma^{2}\right)$ & 29.2 & 62.6 & 127.5 & 195.1 \\
$D\left(\sigma^{2} \tau^{-1}\right)$ & 0.02 & 0.0074 & 0.0023 & 0.0012
\end{tabular}

${ }^{a} R_{\mathrm{e}}$ is the average end-to-end vector magnitude. $D$ is the diffusivity.

- $\left.\left(r / R_{0}\right)^{2}\right)$, which constrains the distance between adjacent monomers to about $1 \sigma$ (we use the same parameters for the FENE potential as Grest and Kremer in refs 5 and 8). We performed constant volume simulations of monodisperse polymer melts of varying chain lengths, and will focus our attention on the crossover regime, specifically $N=20,40,80$ and 120 , respectively. In a typical simulation we use a total of 2400 monomers embedded in the periodic simulation box, of size $14.1 \sigma$ in each direction, which corresponds to the reduced segment density of $\rho^{*}=0.85$. In a few simulations we have doubled the number of monomers, and for the longest chain system, $N=120$, this corresponds to increasing the number of chains from 20 to 40 . Since the properties deduced from the simulation were independent of system size even for these relatively small systems, we conclude that our results only have minor finite size effects. We will report all quantities in terms of reduced units, which are defined at the end of the paper.

We conduct MD simulations using a fifth order Gear algorithm in the microcanonical ensemble with a $\delta t=$ $0.001 t^{*}$ ensuring energy conservation to within $0.5 \%$ over the whole constant energy simulation run. The starting structures for our runs were constructed by gradually squeezing semidilute solutions to a final density of 0.85 over $\sim 10$ million MD steps at the reduced temperature, $T^{*}=1$. These structures were further run at constant temperature, for an additional 10 million steps to obtain a starting configuration for the constant energy run. After the structure preparation and equilibration, we followed with the constant energy simulation for a minimum of 50 million MD steps for $N=20$, up to 300 million MD steps for $N=120$. The reduced temperature exhibited small (several percent) fluctuations around unity since we did not couple our simulations to a heat bath.

It is important to check our simulation results for finite-size effects. Kremer and Grest suggest that for these chain lengths a minimum number of 20 chains is required to avoid such effects. Our systems contain 2400 monomers, i.e., 20 chains for $N=120$. In Table 1 below we have tabulated various quantities like the end-toend vector and diffusivity, for all the chain lengths. In particular, the diffusivity, $D$, changed from 0.0012 to 0.0011 , when the system size was doubled from 2400 to 4800 monomers. Since D is typically the most sensitive to finite size effects, and since these $D$ values closely track the results of Kremer and Grest, ${ }^{5}$ we conclude that our system sizes are large enough.

To check for equilibration we use the autocorrelation function of the end-to-end vector of the chains, ${ }^{4}$ $\left\langle r_{\text {end }}(t) r_{\text {end }}(0)\right\rangle\left\langle\left\langle r_{\text {end }}{ }^{2}\right\rangle\right.$, where $r_{\text {end }}(t)$ denotes the end-to-end distance vector at time $t$. A similar autocorrelation function can also be defined for the root-mean-square radius of gyration. Both of these autocorrelation functions showed an exponential decay, from which the

\section{8} 59 60 61 62 63 64 65 66 67 68 69 70 71 72 73 74 75 76 77 78 79 80 81 82 83 84 85 86 87 88 89 90 91 92 93 94 95 96 97 98 99 


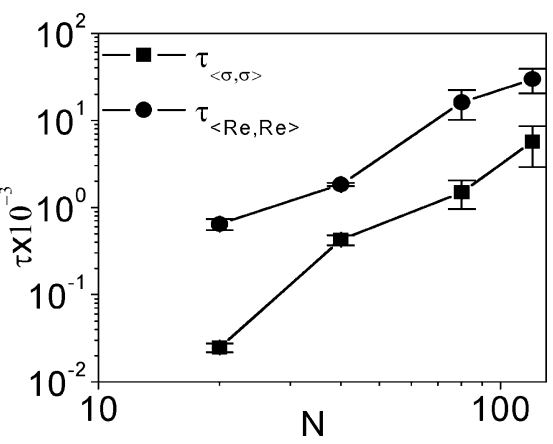

Figure 1. Relaxation time from the stress relaxation and endto-end vector relaxation as a function of chain length.

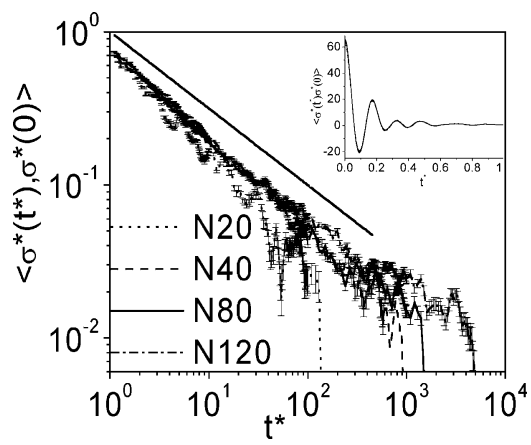

Figure 2. Stress autocorrelation function of the total stress. We stress that the intermediate time behavior of this function corresponds to a $t^{-1 / 2}$ scaling expected from the Rouse model (dark line), while the long time decay is well described by an exponential. Inset shows the initial part showing the short time scale fluctuations arising from bond interactions.

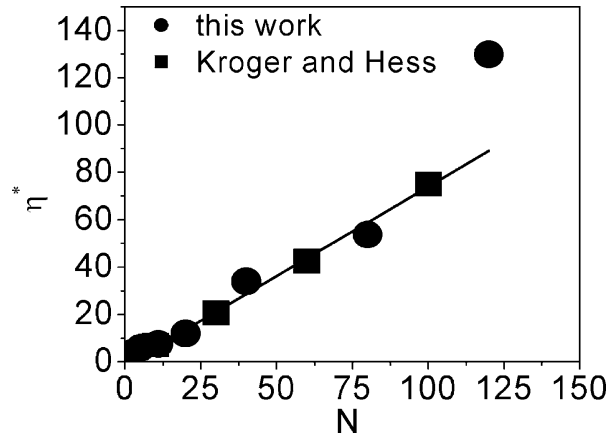

Figure 3. Plot of viscosity as a function of chain length. The line is a best fit to the viscosity for all $N \leq 100$. equilibration time of these properties is readily obtained. Figure 1 shows that, as expected, the relaxation time of the end-to-end distance vector increases with increasing chain length. The trends from the end-to-end vector and stress relaxation seem to be different for $N=20$, but the two curves track each other for all longer chains.

Calculation of the zero shear rate viscosity using the Green-Kubo formula requires the stress autocorrelation function (acf). The stress acfs are plotted in Figure 2. We calculate the stresses through the use of the atomic virial defined as $\sigma_{i j}=(1 / V)\left[-n k_{\mathrm{B}} T \delta_{i j}+\right.$ $\left.\sum_{\alpha}\left(r_{\alpha}{ }^{-1} U_{\alpha}^{\prime}\left(r_{\alpha}\right) r_{\alpha i} r_{\alpha j}\right)\right]$. where $\sigma_{i j}$ is the stress for atom pair $i j$. The first term is the kinetic energy contribution while the second term accounts for all bonded and nonbonded interactions ( $\alpha$ is thus a summation over all atom pairs in the system). Comparison of our approach with existing literature (see Figure 3) suggests that our methodology is accurate for the calculation of transport properties. The acfs of the three off-diagonal elements of the stress tensor are expected to be equivalent due to the isotropy of the system, and hence these are averaged to improve their signal. Even after several hundred million simulation steps, the resulting correlation functions are found to be noisy. The most common procedure for noise reduction in stochastic processes is averaging over several independent data sets. However, we found that beyond a certain threshold, additional averaging does not reduce the noise. The signal at this point is still very noisy, which indicates that the intrinsic noise level in the system is very high. We suspect the noise arises from numerical precision issues, at the small magnitudes of the stress correlation function, especially at long times. So a running average was performed. This average for each time $t$, was defined as the average from $0.9 t$ to $1.1 t$. This procedure preserves the fine features of the correlation function at short times and at the same time significantly reduces the noise at large times. Additionally, since the data for the two different system sizes for $N=120$ were found to superpose we additionally averaged them to improve the signal-to-noise concerns.

Results. In Figure 2 (inset) we see that the stress acf has short time, oscillatory behavior. This is attributed primarily to the rapid fluctuations of the stiff bond potential. The long time behavior arises from the Rouse like dynamics of the single chain and any chainchain interactions. In particular, the intermediate time scale behavior of the stress acf is consistent with the Rouse model scaling, $t^{-1 / 2}$, while the long time decay is exponential, with a time constant characterizing the longest stress relaxation time in the system. It is clear that the longest relaxation time from the stress autocorrelation function (see Figure 1) follows the same power law scaling with $N$ as the end-to-end vector relaxation time at least for $N \geq 40$. However, surprisingly, the stress relaxation time is about an order of magnitude smaller than the end-to-end distance relaxation time. This result may be a consequence of the fact that the end-to-end acf only decays to zero when the orientations trudecorrelate in space (i.e., both in magnitude and orientation), while the stress correlation function is related to only that fraction of stress that has remained unrelaxed. We conclude that orientational decorrelation may be too restrictive a requirement for stress relaxation.

The viscosity is obtained using the Green-Kubo relationship: $\eta^{0}=\left(V / k_{\mathrm{B}} T\right) \int_{0}^{+\infty}\left\langle\mathrm{s}_{x y}(t) \sigma_{x y}(0)\right\rangle \mathrm{d} t$. It is plotted as a function of $N$ in Figure 3. Our results are in good agreement with the data from Kroger and Hess ${ }^{11}$ who performed NEMD simulations over a range of strain rates, and extrapolated to the zero shear limit. However, it should be noted that their system had a density, $\rho^{*}=0.84$, while the density of our system is $\rho^{*}$ $=0.85$. Note that the viscosity appears to vary linearly with $N$ for $N \leq 100$, while the $N=120$ simulation has a distinctly larger viscosity. On this basis one might argue that the first dynamic manifestations of entanglement are only felt for $N>100$ : we shall discuss this point further below.

The stress acf is also used to generate the complex, frequency-dependent modulus of the melt. The relation between the moduli and the stress acf is

$$
\begin{aligned}
& G^{*}(\omega)=G^{\prime}(\omega)+i G^{\prime \prime}(\omega)= \\
& \quad i \omega \frac{V}{k_{\mathrm{B}} T} \int_{0}^{+\infty} e^{-i \omega} t\left\langle\sigma_{x y}(t) \sigma_{x y}(0)\right\rangle \mathrm{d} t
\end{aligned}
$$


Macromolecules
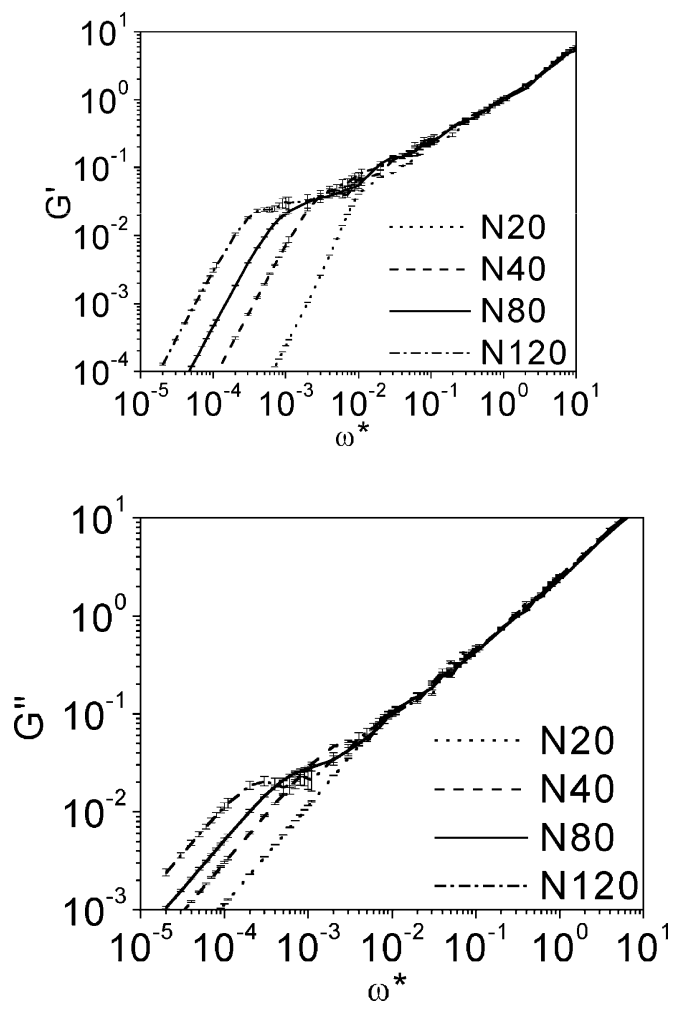

Figure 4. Storage and loss modulii as a function of reduced frequency for different chain length melts.

We plot $G^{\prime}(\omega)$ and $G^{\prime \prime}(\omega)$, the storage and loss modulus, respectively, as a function of reduced frequency, $\omega^{*}$, in Figure 4. Both moduli were obtained via a numerical integration of the acfs, which have been smoothed by the running average procedure described above. For $N$ $\geq 80$, we find that $G^{\prime}(\omega)$ shows evidence for an entanglement plateau, a result which is even clearer for the $N$ $=120$. The plateau modulus for $N=120$ is found to be $G_{N}^{0}=0.03$. The entanglement chain length can then be estimated from the theory of rubber elasticity: $N_{\mathrm{e}, \mathrm{p}}=$ $\left(\rho k_{\mathrm{B}} T / G_{N}^{0}\right)$. We find $N_{\mathrm{e}, \mathrm{p}}=28$. This number is close to the value of 32 obtained by Kremer and Grest from diffusion coefficient and also the relaxation times of the melts. ${ }^{5,8}$ However, NEMD simulations report a much higher value for the $N_{\mathrm{e}} \approx 80$, but Kremer and Grest suggest that these higher numbers might only be obtained for much longer chains. Previous experimental results of Richter, ${ }^{12}$ using neutron spin-echo studies of the dynamic structure factor, report an $N_{\mathrm{e}}=30$. The precise definition of the entanglement length is thus unresolved, and we argue that this matter can be addressed only by conducting equilibrium simulations on truly long chains.

Discussion. Two issues bear more discussion. First, the two different estimates for the entanglement molecular weight obtained from our simulations and those of Kremer and Grest need to be reconciled. Second, we need to understand why the viscosity appears to vary linearly with chain length even in the regime where the chains are clearly longer than the entanglement threshold.

To understand the second point, we decompose the viscosity into three components: (i) the early time oscillatory contribution; (ii) the Rouse contribution where the stress relaxation varies inversely with the square root of time; (iii) the exponential contribution that governs the longest relaxation time of the stress

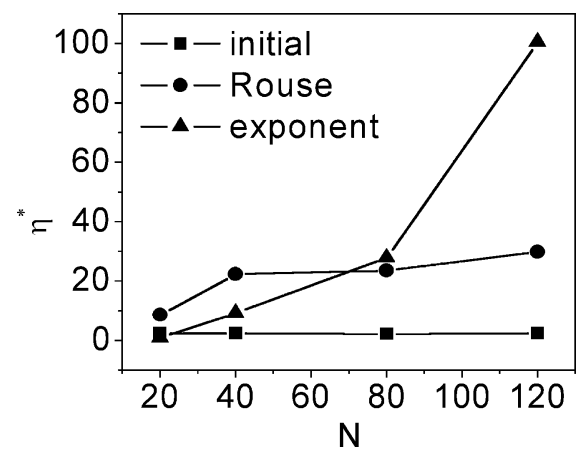

Figure 5. Different contributions to the viscosity as discussed in the text.

acf. Figure 5 shows these three contributions as a 234 function of chain length. We find that the contribution 235 from the first section is small and independent of $N$ as 236 expected, because it comes from the bond vibrations and 237 is hence independent of chain length. The Rouse con- 238 tribution to the viscosity varies linearly with $N$, again, 239 as expected for short chains. But its percentage contri- 240 bution to the total viscosity decreases, since the contri- 241 bution from the longest relaxation time increases strongly 242 with $N$. Note that the Rouse contribution appears to 243 plateau above $N=40$, clearly signaling the addition of 244 extra mechanisms to the dynamics, beyond simple 245 Rouse. This is consistent with our estimate of an 246 entanglement length of 28, and also with the notion that 247 chain motion is only Rouse like between entanglement 248 points. The steep scaling of viscosity, beyond the linear 249 regime, clearly comes from the terminal drop-off region. 250 Even though the contribution from the longest relax- 251 ation time increases strongly with $N$, it only becomes 252 comparable to the Rouse contribution at a chain length 253 of $N=80$. Thus, even though the chains are com- 254 pletely out of the Rouse regime and in the entangled 255 region by that chain length, one still has to go to much 256 higher chain lengths before the viscosity scaling with 257 chain length is perceptibly different from the Rouse 258 scaling.

Next we focus on resolving the apparent discrepancies between the different simulation estimates of the entanglement chain length. First, our estimates of the entanglement length are consistent with those derived from the crossover of the diffusion scaling from the Rouse regime to reptation reported previously by Kremer and Grest. ${ }^{8}$ Since these estimates are derived from relatively short chain lengths, it is reassuring that they agree. In contrast, the simulations of Grest and Kremer yield estimates for the elastic modulus of truly long chains $(N>350)$ which are about a factor of $2-3$ smaller than our plateau values. Consequently, Kremer and Grest find larger values for the entanglement length. While these results would suggest that a very strong finite chain size effect is in play here, as has been conjectured by Kremer and Grest, ${ }^{8}$ experimental results suggest that the plateau modulus, if anything, increases with increasing chain length. On this basis, we conjecture that our estimate is an underestimate rather than overestimate the value of the plateau for longer chains. ${ }^{13}$ We point to the fact that the Kremer-Grest estimates were from simulations where the melt is initially stretched by a large amount and then allowed to relax to equilibrium. It is unclear to us if these results yield the zero shear storage modulus corresponding to the entanglement plateau or if these estimates correspond to the shear thinning regime. If these simulations were 
in the shear thinning regime, as might be expected from the simulation of Kroger and Hess, ${ }^{12}$ then the resulting elastic plateau moduli would be too small, thus resulting in high estimates of the entanglement chain length. With all these points, it is appropriate to conclude that the numerical value of entanglement length is only known to certainty to within a factor of 2 : more conclusive equilibrium MD simulations for much longer chains are necessary, and are currently being conducted, to unequivocally resolve these issues.

Conclusions. We have conducted equilibrium MD simulations to determine the stress relaxation of polymer melts in the crossover regime between Rouse and reptation, and find that the storage modulus and the stress autocorrelation function appear to show signatures consistent with reptation dynamics, especially for chains longer than $N=80$. We estimate an entanglement chain length of 28 from the value of the storage modulus plateau, which is consistent with previous estimates from equilibrium chain diffusion simulations and experiment, but not with more recent nonequilibrium simulations of Kremer and Grest. The entanglement length is thus only known to within a factor of 2 . A surprising result, which is potentially important for the simulation of the mechanical properties of polymer melts, is that the longest relaxation time for the stress acf is several times smaller than those estimated from acf of structural quantities, such as the end-to-end distance acf.

Acknowledgment. We thank Gary Grest for useful discussions. Financial support for this work was provided by the Office of Naval Research Contract No. N00014-01-10732 (P.K.; S.K.K.), the National Science Foundation (DMR-01xxx; S.K.K.), and the National
Science Foundation Nanoscale Science and Engineering 321 Center at RPI, NSF Grant No. DMR-0117792 (S.K.K.; 322 P.K.).

\section{Nomenclature}

length parameter: $\sigma$ well depth in LJ interaction: $\epsilon$

reduced time: $t^{*}=t / \tau ; \tau=\sigma \sqrt{m / \epsilon}$

reduced temperature: $T^{*}=\left(k_{\mathrm{B}} T / \epsilon\right)$

reduced density: $\rho^{*}=0.85=\rho \sigma^{3}$

reduced viscosity: $\eta^{*}=\eta / k_{\mathrm{B}} T \sigma^{-3} \tau$ $k_{\mathrm{B}} T \sigma^{-3}$

reduced plateau modulus: $G_{\mathrm{N}}^{0}=G_{\mathrm{N}} / k_{\mathrm{B}} T \sigma^{-3}$ reduced frequency: $\omega^{*}=\omega \tau$

\section{References and Notes 335}

(1) de Gennes, P. G. Scaling Concepts in Polymer Physics; 336 Cornell University Press: Ithaca, NY, 1979.

(2) Doi, M.; Edwards, S. F. The Theory of Polymer Dynamics; 338 Clarendon: Oxford, U.K., 1986.

(3) Kreer, T.; Baschnagel, J.; Muller, M.; Binder, K. Macromol- 340 ecules 2001, 34, 1105.

(4) Paul, W.; Binder, K.; Heermann, D. W.; Kremer, K. J. Chem. 342 Phys. 1991, 95, 7726.

J. Chem Phys 1990, $92,5057$.

(6) Padding, J. T.; Briels, W. J. J. Chem. Phys. 2002, 117, 925. 345

(7) Everaers, R.; Sukumaran, S. K.; Grest, G. S.; Svaneborg, 346 C.; Sivasubramian, A.; Kremer, K. Science 2004, 303, 823. 347

(8) Putz, M.; Kremer, K.; Grest, G. Europhys. Lett. 2000, 49, 348 735.

(9) Gao, J.; Weiner, J. H. J. Chem. Phys. 1995, 103, 1621 and 350 references therein.

(10) Bedrov, D.; Smith, G. D.; Douglas, J. F. Europhys. Lett. 352 2002, 59, 384

(11) Kroger, M; Loose, W; Hess, S. J. Rheol. 1993, 37, 1057.

(12) Wischenwski, A.; Richter, D. Europhys. Lett. 2000,52, 719.355

(13) Onogi, S.; et. al. Macromolecules 1970, 3, 109.

MA035487L 